Article

\title{
Antimicrobial Activity of Zabofloxacin against Clinically Isolated Streptococcus pneumoniae
}

\author{
Hee-Soo Park ${ }^{1,+}$, Sang-Hun Oh ${ }^{2,+}$, Hye-Shin Kim ${ }^{2}$, Dong-Rack Choi ${ }^{3}$ and Jin-Hwan Kwak ${ }^{2, *}$ \\ 1 School of Food Science and Biotechnology, Kyungpook National University, Daegu 41566, Korea; \\ phsoo97@knu.ac.kr \\ 2 School of Life Science, Handong Global University, 558 Handong-ro, Buk-gu, Pohang 37554, Korea; \\ osh8755@naver.com (S.-H.O.); momobile217@gmail.com (H.S.K.) \\ 3 Dong Wha Pharm., Ind. Co. Ltd., Anyang 31041, Korea; dongrack.choi@dong-wha.co.kr \\ * Correspondence: jhkwak@handong.edu; Tel.: +82-54-260-1353; Fax: +82-54-260-1925 \\ + These authors contributed equally to this work.
}

Academic Editor: Diego Muñoz-Torrero

Received: 15 September 2016; Accepted: 9 November 2016; Published: 17 November 2016

\begin{abstract}
Zabofloxacin is a novel fluoroquinolone agent that has potent activity against gram-positive pathogens. In this study, we confirmed that zabofloxacin showed the most potent in vitro and in vivo activities against drug-resistant Streptococcus pneumoniae. Among the fluoroquinolone compounds, zabofloxacin showed the most potent in vitro activity against clinical isolates of penicillin-sensitive S. pneumoniae (minimum inhibitory concentration, $\mathrm{MIC}_{90}: 0.03 \mathrm{mg} / \mathrm{L}$ ) and penicillin-resistant S. pneumoniae ( $\mathrm{MIC}_{90}: 0.03 \mathrm{mg} / \mathrm{L}$ ). Against quinolone-resistant S. pneumoniae, zabofloxacin ( $\mathrm{MIC}_{90}: 1 \mathrm{mg} / \mathrm{L}$ ) was more active than ciprofloxacin, sparfloxacin, and moxifloxacin; however, its activity was the same as that of gemifloxacin. The in vivo activity of zabofloxacin was most potent among the quinolone compounds tested against the systemic infection and respiratory tract infection models in mice.
\end{abstract}

Keywords: zabofloxacin; minimum inhibitory concentration; Streptococcus pneumoniae; in vivo

\section{Introduction}

Streptococcus pneumoniae is one of the most important pathogenic bacteria known to cause community-acquired pneumonia, acute otitis media, and meningitis, with high morbidity and mortality rates [1]. Various antibiotics including beta-lactams and macrolide agents are used to treat bacterial pneumonia; however, the emergence of antibiotic-resistant bacteria has been rapid worldwide [2]. In the United States, $30 \%$ of S. pneumoniae strains are resistant to one or more antibiotics including penicillin, other beta-lactams, and macrolide agents [3,4]. Therefore, fluoroquinolones and ketolides have been considered for the treatment of multi-drug resistant pneumococci [5].

Zabofloxacin (DW-224a) is a novel fluoroquinolone antibiotic with potent antibacterial activity against gram-positive cocci [6,7]. Previous studies demonstrated that zabofloxacin had effective in vitro activity against drug-resistant $S$. pneumoniae isolates that caused non-invasive and invasive disease [6,8,9]. In this study, we further examined the in vitro activities of zabofloxacin against quinolone-susceptible (QSSP) and quinolone-resistant S. pneumoniae (QRSP) strains when compared to other antimicrobial agents. Moreover, we found that zabofloxacin was the most potent antibacterial agent against penicillin-resistant S. pneumoniae (PRSP) in the murine systemic infection model used in this study. 


\section{Results}

The minimum inhibitory concentrations (MICs) of compounds tested against QSSP are presented in Table 1. The $\mathrm{MIC}_{90}$ of zabofloxacin was $0.03 \mathrm{mg} / \mathrm{L}$ ( $\mathrm{MIC}_{90}$ is the concentration at which $90 \%$ of the strain growth is inhibited), which is the lowest among the compounds tested, followed by gemifloxacin ( $\mathrm{MIC}_{90}: 0.03 \mathrm{mg} / \mathrm{L}$ ), moxifloxacin $\left(\mathrm{MIC}_{90}: 0.25 \mathrm{mg} / \mathrm{L}\right.$ ), sparfloxacin ( $\mathrm{MIC}_{90}: 0.5 \mathrm{mg} / \mathrm{L}$ ), and ciprofloxacin ( $\left.\mathrm{MIC}_{90}: 2 \mathrm{mg} / \mathrm{L}\right)$. QSSP strains were also found to be susceptible to linezolid $\left(\mathrm{MIC}_{90}: 1 \mathrm{mg} / \mathrm{L}\right.$ ) and synercid (MIC $90: 1 \mathrm{mg} / \mathrm{L}$ ). These results indicated that zabofloxacin was most potent among the agents tested against QSSP strains.

Against 22 QRSP strains (ciprofloxacin MICs $\geq 8.0 \mathrm{mg} / \mathrm{L}$ ) that contain a mutation in the quinolone resistance-determining region (QRDR), zabofloxacin (MIC range: 0.06-2 mg/L; $\mathrm{MIC}_{90}: 1.0 \mathrm{mg} / \mathrm{L}$ ) was more active than ciprofloxacin (MIC range: 4-64 mg/L; $\mathrm{MIC}_{90}: 64.0 \mathrm{mg} / \mathrm{L}$ ) and moxifloxacin (MIC range: $4-8 \mathrm{mg} / \mathrm{L} ; \mathrm{MIC}_{90}: 8.0 \mathrm{mg} / \mathrm{L}$ ), and as active as gemifloxacin (MIC range: $0.06-2 \mathrm{mg} / \mathrm{L}$; $\mathrm{MIC}_{90}: 1.0 \mathrm{mg} / \mathrm{L}$ ) (Table 2). All 22 strains contain 2 or more mutations in the QRDR of gyrA, parC, and/or parE, but not gyrB (Table 3). In the presence of reserpine, an efflux pump inhibitor [10], 3 out of 22 strains exhibited MIC lower than that of ciprofloxacin and 1 strain exhibited MIC lower than that of gemifloxacin. However, all isolates showed the same MIC for zabofloxacin and moxifloxacin in the presence of an efflux inhibitor (Table 3). These results demonstrated that zabofloxacin is effective against mutation in the QRSP of target genes and a poor substrate for efflux pumps.

To examine the bactericidal activity of zabofloxacin against S. pneumoniae, time-kill analysis was carried out. As shown in Figure 1, zabofloxacin and gemifloxacin showed rapid bactericidal activity at 2 and 4 times the MIC against QSSP and QRSP. Ciprofloxacin showed bactericidal activity at 4 times MIC against QRSP only, but this concentration of ciprofloxacin $(128 \mathrm{mg} / \mathrm{L})$ could not be used for the treatment of S. pneumoniae. The regrowth of tested strains was completely inhibited by zabofloxacin.

A

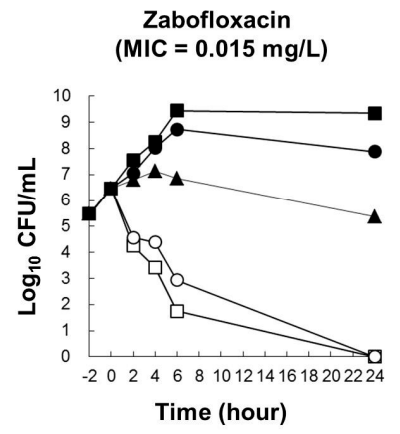

B

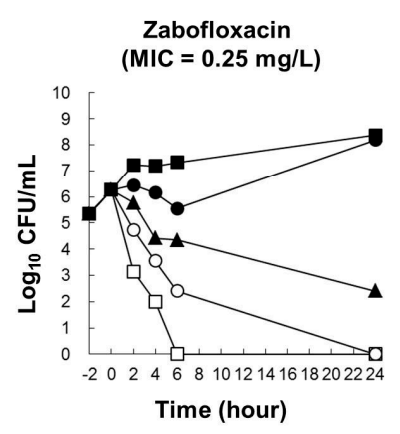

S. pneumoniae 18 (QSSP)
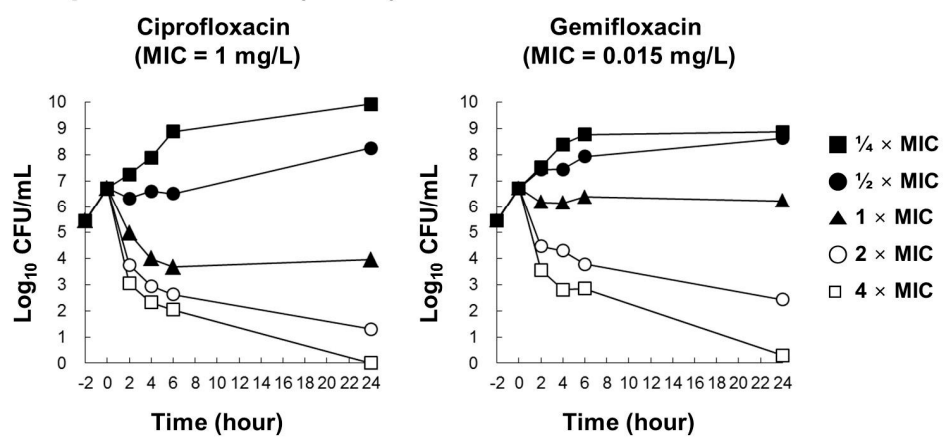

S. pneumoniae 107,282 (QRSP)

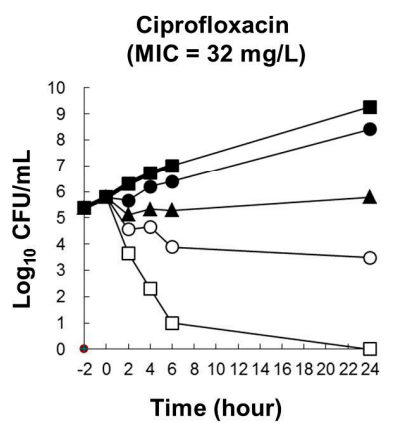

Figure 1. Time-kill curves of zabofloxacin, ciprofloxacin, and gemifloxacin against Streptococcus pneumoniae. (A) S. pneumoniae 18 (quinolone-susceptible S. pneumoniae, QSSP) exposed to zabofloxacin, ciprofloxacin, and gemifloxacin; (B) S. pneumoniae 107,282 (quinolone-resistant S. pneumoniae, QRSP) exposed to zabofloxacin, ciprofloxacin, and gemifloxacin. 
Table 1. Agar dilution minimum inhibitory concentrations (MICs) of 84 quinolone-susceptible Streptococcus pneumoniae strains (with ciprofloxacin MICs <4.0 mg/L).

\begin{tabular}{|c|c|c|c|c|c|c|c|c|c|c|c|c|c|c|c|c|c|}
\hline \multirow{2}{*}{$\begin{array}{c}\text { Organisms } \\
\text { (No. of Organisms) }\end{array}$} & \multirow{2}{*}{ Compounds } & \multirow{2}{*}{$\mathrm{MIC}_{90}{ }^{\mathrm{a}}$} & \multicolumn{15}{|c|}{ No. of Isolates with the Following MIC (mg/L) } \\
\hline & & & 0.008 & 0.015 & 0.03 & 0.06 & 0.125 & 0.25 & 0.5 & 1 & 2 & 4 & 8 & 16 & 32 & 64 & $>64$ \\
\hline \multicolumn{18}{|l|}{ PSSP $^{b} \&$ PISP $^{c}(27)$} \\
\hline & Zabofloxacin & 0.03 & 8 & 8 & 11 & & & & & & & & & & & & \\
\hline & Ciprofloxacin & 2 & & & & & & & 8 & 10 & 9 & & & & & & \\
\hline & Sparfloxacin & 0.5 & & & & 3 & 7 & 8 & 9 & & & & & & & & \\
\hline & Moxifloxacin & 0.25 & & & 1 & 8 & 9 & 9 & & & & & & & & & \\
\hline & Gemifloxacin & 0.03 & 8 & 5 & 13 & 1 & & & & & & & & & & & \\
\hline & Penicillin G & 1 & 1 & 3 & 5 & & 1 & 3 & 7 & 7 & & & & & & & \\
\hline & Oxacillin & 8 & 2 & 1 & 1 & 4 & 1 & 2 & 3 & 5 & & 1 & 5 & 2 & & & \\
\hline & Erythromycin & $>64$ & 3 & 3 & 6 & & & & 2 & & 2 & 2 & & & & & 9 \\
\hline & Linezolid & 1 & & & & & & 5 & 13 & 9 & & & & & & & \\
\hline & Synercid & 1 & & & & & 1 & 11 & 2 & 13 & & & & & & & \\
\hline \multicolumn{18}{|l|}{ PRSP d $^{d}(57)$} \\
\hline & Zabofloxacin & 0.03 & 8 & 30 & 19 & & & & & & & & & & & & \\
\hline & Ciprofloxacin & 2 & & & & & & & 4 & 29 & 24 & & & & & & \\
\hline & Sparfloxacin & 0.5 & & & & 1 & 9 & 27 & 20 & & & & & & & & \\
\hline & Moxifloxacin & 0.25 & & & & 5 & 29 & 23 & & & & & & & & & \\
\hline & Gemifloxacin & 0.03 & 9 & 17 & 27 & 4 & & & & & & & & & & & \\
\hline & Penicillin G & 4 & & & & & & & & & 27 & 30 & & & & & \\
\hline & Oxacillin & 16 & & & & & & & & & 1 & 8 & 14 & 32 & 2 & & \\
\hline & Erythromycin & $>64$ & & & 2 & 1 & & 1 & 4 & 8 & 2 & & 2 & & & 3 & 34 \\
\hline & Linezolid & 1 & & & & & & 10 & 17 & 30 & & & & & & & \\
\hline & Synercid & 2 & & & & & & 20 & 13 & 18 & 6 & & & & & & \\
\hline
\end{tabular}
S. pneumoniae.

Table 2. MICs of fluoroquinolones against 22 fluoroquinolone-resistant strains.

\begin{tabular}{cccc}
\hline Quinolone & MIC Range & MIC $_{\mathbf{5 0}}{ }^{\text {a }}$ & MIC $_{\mathbf{9 0}}$ \\
\hline Zabofloxacin & $0.06-2$ & 0.25 & 1 \\
Ciprofloxacin & $4-64$ & 32 & 64 \\
Moxifloxacin & $2-8$ & 4 & 8 \\
Gemifloxacin & $0.06-2$ & 0.25 & 1 \\
\hline
\end{tabular}

${ }^{\mathrm{a}} \mathrm{MIC}_{50}$ : MIC at which $50 \%$ of the strains are inhibited. 
Table 3. MICs for 22 Streptococcus pneumoniae strains with defined mutations in the quinolone resistance-determining regions (QRDRs).

\begin{tabular}{|c|c|c|c|c|c|c|c|}
\hline \multirow{2}{*}{ Strain No. } & \multicolumn{4}{|c|}{ MIC (mg/L) } & \multicolumn{3}{|c|}{ Detected Mutation(s) in QRDRs } \\
\hline & $\mathrm{ZAB}^{\mathrm{a}}$ & $\mathrm{CIP}{ }^{\mathrm{b}}$ & $\operatorname{MOX}^{c}$ & GEM $^{d}$ & gyrA & parC & parE \\
\hline 104340 & 0.06 & 4 & 2 & 0.06 & E85K & K136T & $\mathrm{D} 435 \mathrm{~N}$ \\
\hline 112519 & 0.125 & 4 & 2 & 0.06 & $\mathrm{~S} 81 \mathrm{~F}$ & $\mathrm{~S} 79 \mathrm{~F}$ & - e \\
\hline 104376 & 0.125 & 4 & 2 & 0.125 & $\mathrm{~S} 81 \mathrm{~F}$ & - & E474K \\
\hline YS2 & 0.06 & 8 & 2 & 0.06 & $\mathrm{~S} 81 \mathrm{~F}$ & - & D435N, I460V \\
\hline YS1 & 0.125 & 8 & 2 & 0.125 & $\mathrm{~S} 81 \mathrm{~F}$ & $\mathrm{D} 83 \mathrm{G}, \mathrm{K} 137 \mathrm{~N}$ & $\mathrm{I} 460 \mathrm{~V}$ \\
\hline 622286 & 0.25 & 8 & 2 & 0.125 & $\mathrm{~S} 81 \mathrm{~F}$ & $\mathrm{D} 83 \mathrm{~N}$ & - \\
\hline 102575 & 0.06 & 16 & 2 & 0.125 & $\mathrm{~S} 81 \mathrm{~F}$ & - & $\mathrm{I} 460 \mathrm{~V}$ \\
\hline 503167 & 0.125 & 16 & 4 & 0.06 & $\mathrm{~S} 81 \mathrm{~F}$ & S79F, K137N & $\mathrm{I} 460 \mathrm{~V}$ \\
\hline 103845 & 0.125 & 16 & 4 & 0.125 & $\mathrm{~S} 81 \mathrm{~F}$ & $\mathrm{~S} 79 \mathrm{~F}$ & - \\
\hline 114794 & 0.125 & 16 & 4 & 0.25 & $\mathrm{~S} 81 \mathrm{~F}$ & S79F & I460V \\
\hline 102830 & 0.125 & 16 & 4 & 0.25 & S81Y & D78N, S79F & - \\
\hline 102182 & 0.125 & 32 & 4 & 0.25 & $\mathrm{~S} 81 \mathrm{~F}$ & $\mathrm{~S} 79 \mathrm{~F}, \mathrm{~K} 137 \mathrm{~N}$ & $\mathrm{I} 460 \mathrm{~V}$ \\
\hline 102239 & 0.25 & 32 & 4 & 0.5 & $\mathrm{~S} 81 \mathrm{~F}$ & S79F & $\mathrm{I} 460 \mathrm{~V}$ \\
\hline 102924 & 0.25 & 32 & 4 & 0.5 & $\mathrm{~S} 81 \mathrm{~F}$ & S79F & - \\
\hline 104710 & 0.25 & 32 & 4 & 0.5 & $\mathrm{~S} 81 \mathrm{~F}$ & $\mathrm{~S} 79 \mathrm{~F}$ & - \\
\hline 107282 & 0.25 & 32 & 4 & 0.25 & $\mathrm{~S} 81 \mathrm{~F}$ & D83Y, K137N & $\mathrm{I} 460 \mathrm{~V}$ \\
\hline 113165 & 0.25 & 32 & 4 & 0.25 & $\mathrm{~S} 81 \mathrm{~F}$ & S79Y, K137N & $\mathrm{I} 460 \mathrm{~V}$ \\
\hline SNU14 & 0.25 & 32 & 4 & 0.25 & $\mathrm{~S} 81 \mathrm{~F}$ & S79F, K137N & $\mathrm{D} 435 \mathrm{~N}, \mathrm{I} 460 \mathrm{~V}$ \\
\hline SNU17 & 0.25 & 32 & 4 & 0.25 & $\mathrm{~S} 81 \mathrm{~F}$ & $\mathrm{~S} 79 \mathrm{~F}, \mathrm{~K} 137 \mathrm{~N}$ & $\mathrm{P} 454 \mathrm{~S}, \mathrm{I} 460 \mathrm{~V}$ \\
\hline 120963 & 1 & $64^{\mathrm{f}}$ & 8 & 1 & E85K & S79F, K137N & - \\
\hline 103709 & 1 & $64^{\mathrm{f}}$ & 8 & 1 & $\mathrm{~S} 81 \mathrm{~F}$ & S79F & - \\
\hline 103672 & 2 & $64^{\mathrm{f}}$ & 8 & $2^{f}$ & E85K & S79F & - \\
\hline
\end{tabular}

Previous studies reported that the zabofloxacin showed the most potent activity against penicillin-susceptible S. pneumoniae (PSSP) [6,7]. In this study, we examined in vivo efficacies of zabofloxacin against systemic infections and compared the results with those of ciprofloxacin, moxifloxacin, and gemifloxacin against PRSP 1065 strain. Zabofloxacin was the most potent quinolone (MIC: $0.015 \mathrm{mg} / \mathrm{L} ; \mathrm{ED}_{50}$ (median effective dose): $0.42 \mathrm{mg} / \mathrm{kg}$ ), followed by ciprofloxacin (MIC: $4 \mathrm{mg} / \mathrm{L} ; \mathrm{ED}_{50}: 31.45 \mathrm{mg} / \mathrm{kg}$ ), moxifloxacin (MIC: $0.25 \mathrm{mg} / \mathrm{L} ; \mathrm{ED}_{50}: 18.00 \mathrm{mg} / \mathrm{kg}$ ), and gemifloxacin (MIC: $0.03 \mathrm{mg} / \mathrm{L} ; \mathrm{ED}_{50}: 2.09 \mathrm{mg} / \mathrm{kg}$ ) (Table 4). These results agreed well with the in vitro MIC values. They indicated that zabofloxacin exhibits the most potent protective effects against systemic infections caused by penicillin-susceptible S. pneumoniae and PRSP. We then examined the in vivo effect using the pneumococcal pneumoniae model. As shown Figure 2, zabofloxacin significantly reduced bacterial counts in the lung compared with moxifloxacin.

Table 4. Comparative in vivo activities of zabofloxacin against systemic infections in mice.

\begin{tabular}{|c|c|c|c|}
\hline Microorganism (Inoculum) & Antimicrobial Agent ${ }^{a}$ & $\mathrm{MIC}(\mathrm{mg} / \mathrm{L})$ & $\begin{array}{c}\mathrm{ED}_{50}(\mathrm{mg} / \mathrm{kg})^{\mathrm{b}} \\
(95 \% \text { Confidence Limits) }\end{array}$ \\
\hline \multirow{4}{*}{ 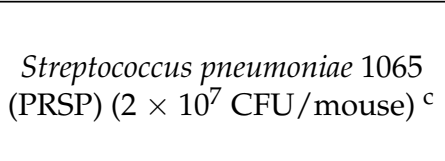 } & Zabofloxacin & 0.03 & $0.42(0.04-1.62)$ \\
\hline & Ciprofloxacin & 4 & $31.45(6.56-999)$ \\
\hline & Moxifloxacin & 0.25 & $18.00(4.44-244)$ \\
\hline & Gemifloxacin & 0.03 & $2.0(0.15-7.99)$ \\
\hline
\end{tabular}

\footnotetext{
${ }^{a}$ Antimicrobial agents were orally administered twice at 1 and $4 \mathrm{~h}$ post infection; ${ }^{\mathrm{b}} \mathrm{ED}_{50}$ : median effective dose
} needed to protect $50 \%$ of the mice; ${ }^{c} \mathrm{CFU}$ : colony-forming units. 


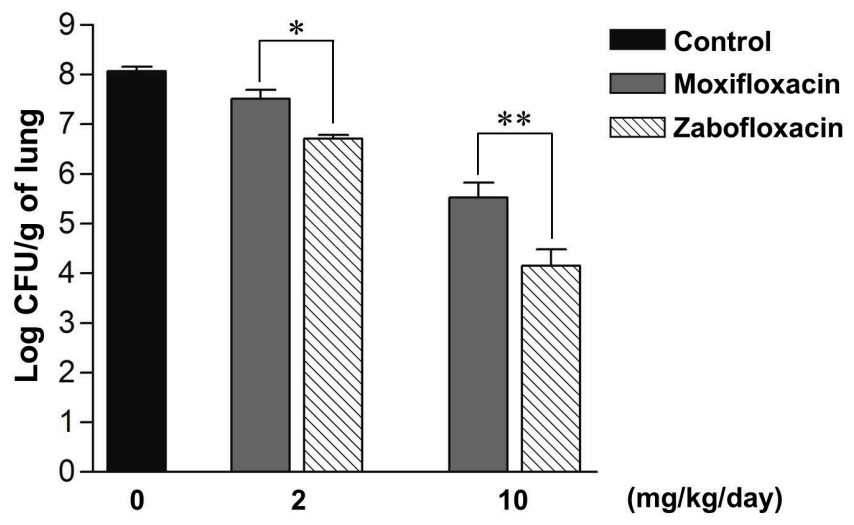

Figure 2. Therapeutic efficacies of zabofloxacin and moxifloxacin in a respiratory tract infection model caused by S. pneumoniae 1065 (PRSP). The number of viable S. pneumoniae in the lungs was examined after infection with PRSP and administration the test drugs. Each bar represents the mean \pm SEM (standard error of the mean) $(n=4)$. Zabofloxacin significantly reduced the number of bacteria compared with moxifloxacin $\left({ }^{*} p<0.05 ;{ }^{* *} p<0.01\right)$.

\section{Discussion}

Zabofloxacin is a novel fluoronaphthyridone quinolone with a 7-pyrrolidone substituent that showed excellent in vitro activities against both gram-positive and gram-negative strains $[6,7]$. Importantly, zabofloxacin has been shown to have excellent in vivo activity against gram-positive pathogens including Steptococcus aureus (S. aureus), Streptococcus pyogenes (S. pyrigenes), and S. pneumonia [6,7]. Zabofloxacin also was very active against pathogenic bacteria that cause community-acquired respiratory tract infections, including Haemophilus influenzae and Moraxella catarrhalis [6,7]. In addition, zabofloxacin could be considered as an alternative candidate for treatment of quinolone-susceptible and quinolone-resistant gonorrhea [11]. Therefore, zabofloxacin is considered a potent antibacterial candidate for clinical trials and has been approved in South Korea for specific treatments $[8,12]$.

Several studies have suggested that zabofloxacin has potent activity against non-invasive and invasive S. pneumoniae [6-8] and has bactericidal activity against several S. pneumoniae strains $[9,13]$. This compound targets DNA gyrase and topoisomerase IV, and mutations in both proteins are needed for the development of high-level resistance in zabofloxacin in S. pneumoniae [14]. In the present study, MIC results further confirmed that zabofloxacin gave the lowest quinolone MICs against ciprofloxacin-susceptible and ciprofloxacin-resistant S. pneumoniae strains (Tables 1-3). Zabofloxacin also had rapid bactericidal activity against PRSP and QRSP (Figure 1). More importantly, the results of in vivo studies demonstrated that zabofloxacin exhibited the most potent protective effects against systemic infection and respiratory tract infection caused by penicillin-resistant S. pneumoniae (Table 4, Figure 2). These results imply that zabofloxacin is useful for clearance of bacteria that grow in the lungs.

A previous pharmacokinetics study has shown that the $C_{\max }$ (maximum serum concentrations), $\mathrm{AUC}_{0-48}$, (the area under the plasma concentration versus time curve (AUC) from the time of dosing to 48 hours post-dosing), and $\mathrm{AUC}_{0-\infty}$ (the AUC extrapolated to infinity) parameters of zabofloxacin hydrochloride were $1.89 \pm 0.49 \mathrm{mg} / \mathrm{L}, 11.11 \pm 2.00 \mathrm{~kg} \cdot \mathrm{h} / \mathrm{L}$, and $11.29 \pm 2.01 \mathrm{~kg} \cdot \mathrm{h} / \mathrm{L}$, respectively. The half-life $\left(t_{1 / 2}\right)$ time of zabofloxacin was $8.2 \pm 1.3 \mathrm{~h} \mathrm{[15].} \mathrm{Analyses} \mathrm{for} \mathrm{phase} 2$ clinical trial dose selection for zabofloxacin proposed that daily doses of $366 \mathrm{mg}$ zabofloxacin provides a probability of fAUC24h/MIC ratio of 30 (free drug divided by the MIC, PK-PD target attainment) is essentially 1.0 for MIC values of $0.03 \mathrm{mg} / \mathrm{L}$ (zabofloxacin MIC 90 against PSSP and PRSP).

While our results demonstrated that zabofloxacin has potent in vitro and in vivo activities against clinical isolated S. pneumoniae, there are some limitations in this study. First, the number of tested isolates is quite small. In addition, the strains were obtained between the years 2001 and 2010 years. Therefore, these epidemiological values in this study cannot represent current epidemiological trends. 


\section{Experimental Section}

\subsection{Antimicrobial Agents}

Zabofloxacin was provided by Dong Wha Pharmaceutical Co. Ltd. (Anyang, Korea). Ciprofloxacin, sparfloxacin, moxifloxacin, linezolid, and synercid were purified from commercial tablets by recrystallization, and determined to be $>99.9 \%$ pure by high-performance liquid chromatography analysis. Gemifloxacin was obtained from LG Chemical Ltd. (Daejeon, Korea). Oxacillin, penicillin G, vancomycin, and erythromycin were purchased from Sigma Aldrich (St. Louis, MO, USA).

\subsection{Bacterial Strains}

For in vitro susceptibility studies, 84 QSSP strains (ciprofloxacin MICs $\leq 4.0 \mathrm{mg} / \mathrm{L}$ ) were obtained from hospitals in Seoul (Korea) between 2001 and 2010. From the collection, we selected 22 QRSP strains (ciprofloxacin MICs $\geq 8.0 \mathrm{mg} / \mathrm{L}$ ) and tested them using the agar dilution MIC test. For the murine systemic infection model, colonies of PRSP 1065 were selected by screening clinical isolates.

\subsection{Antimicrobial Susceptibility Test}

The MICs were determined by the two-fold agar dilution method as described in the guidelines of the Clinical and Laboratory Standards Institute (CLSI) [16]. In brief, test strains were grown for $18 \mathrm{~h}$ in Todd-Hewitt broth (THB, Difco, Detroit, MI, USA) supplemented with $0.5 \%$ yeast extract (Difco) for $18 \mathrm{~h}$ at $37^{\circ} \mathrm{C}$, and then diluted with the same fresh medium to a density of $10^{7}$ colony-forming units (CFU) per milliliter. Cultures were inoculated in Muller-Hinton agar (MHA, Difco) plates supplemented with $5 \%$ defibrinated sheep blood (Komed, Sungnam, Korea) containing serial dilutions of the antimicrobial agents using a multi-pin inoculator to yield $10^{4} \mathrm{CFU} /$ spot. Plates were incubated at $35{ }^{\circ} \mathrm{C}$ for $18 \mathrm{~h}$, and were examined for growth. S. pneumoniae ATCC 6305 was used as a control strain. The MIC was considered the lowest concentration at which growth on agar plates is completely inhibited, disregarding a single colony or a faint haze caused by the inoculum.

\subsection{Determination of Resistance Mechanism}

To examine the efflux mechanism, quinolone-resistant strains were inoculated on agar plates in the presence or absence of $10 \mathrm{mg} / \mathrm{L}$ reserpine (Sigma), as described previously [17]. By definition, an efflux mechanism exists when there is at least a 4-fold lower MIC in the presence of reserpine [17].

QRDR sequences in $g y r A$, gyrB, parC, and parE were amplified by polymerase chain reaction (PCR) using primers described previously [18]. PCR products were purified using AccuPrep ${ }^{\text {TM }}$ PCR Product Purification Kit (Bioneer Co. Ltd., Daejeon, Korea), and then sequenced using a system from Solgent Co. Ltd. (Daejeon, Korea).

\subsection{Time-Kill Analysis}

The time-kill studies were performed using the CLSI M26-A method [19]. In brief, S. pneumoniae 18 (QSSP) and S. pneumoniae 107282 (QRSP) strains were cultured in Muller-Hinton II broth (MHIIB, BD, Sparks, MD, USA) for $18 \mathrm{~h}$ at $37^{\circ} \mathrm{C}$. The cultured microbes were diluted with fresh MHIIB to a density of $10^{5}$ to $10^{6} \mathrm{CFU} / \mathrm{mL}$ and pre-incubated for $2 \mathrm{~h}$. Then, zabofloxacin, ciprofloxacin, and gemifloxacin were added to the cultures at concentrations of $0.25 \times, 0.5 \times, 1 \times, 2 \times$, and $4 \times$ MIC. The numbers of colony forming cells were quantified after $0,2,4,6$ and $24 \mathrm{~h}$ of incubation at $37^{\circ} \mathrm{C}$ for $18 \mathrm{~h}$ by serial dilution on MHA. The compounds were considered bactericidal at the concentration that reduced the original inoculum by $3 \log \mathrm{CFU} / \mathrm{mL}(99.9 \%)$ for each of the time periods.

\subsection{Systemic Infection Model in Mice}

Mice studies were performed as described previously [18]. S. pneumoniae strain was cultured in tryptic soy agar medium (Difco) supplemented with $5 \%$ defibrinated sheep blood at $35{ }^{\circ} \mathrm{C}$ for $18 \mathrm{~h}$. 
For inoculation, S. pneumoniae 1065 was suspended in $0.9 \% \mathrm{NaCl}$. Groups of 6 male ICR mice (Dae Han Bio Link Co. Ltd., Eumseong-gun, Korea, weighing 18-22 g) were challenged intraperitoneally with $0.5 \mathrm{~mL}$ of the bacterial suspension, corresponding to an inoculum ranging from 10 to 100 times the minimal lethal dose (MLD) of the bacteria. Four dose levels were used for each antibiotic, depending on the in vitro antimicrobial activity of the compound. Zabofloxacin, ciprofloxacin, gemifloxacin, and moxifloxacin were administered orally to mice twice at 1 and $4 \mathrm{~h}$ post infection. Mice were housed in animal rooms maintained at $23 \pm 2{ }^{\circ} \mathrm{C}$ with $55 \% \pm 20 \%$ relative humidity. Mortality was recorded for 7 days, and the median effective dose needed to protect $50 \%$ of the mice $\left(E_{50}\right)$ was calculated by the Probit method [20]. The challenge inoculum was sufficient to kill $100 \%$ of the untreated control mice, which died within $48 \mathrm{~h}$ post infection. Experimental protocols were approved by the Ethics Review Committee for Animal Experimentation at Handong Global University (Korea) (protocol \#HGU-2008-01).

\subsection{Respiratory Tract Infection Model in Mice}

Penicillin-resistant S. pneumoniae 1065 strain was cultured in tryptic soy agar medium supplemented with $5 \%$ defibrinated sheep blood at $35{ }^{\circ} \mathrm{C}$ for $18 \mathrm{~h}$. This strain was suspended in $0.9 \% \mathrm{NaCl}$. Male ICR mice (Dae Han Bio Link, weighing 18-22 g) were infected by intranasal route with $20 \mu \mathrm{L}$ of S. pneumoniae 1065 suspension at a dose of approximately $10^{7} \mathrm{CFU} /$ mouse. One day after the inoculation, the animals (in groups of four mice each) were treated with zabofloxacin or moxifloxacin orally once a day at a dose of 2 or $10 \mathrm{mg} / \mathrm{kg}$ for 3 consecutive days. The number of bacteria in the lungs was examined on the day following the final administration of the test drugs, namely, 4 days after inoculation. The lungs were removed aseptically and weighed, and then the viable bacterial counts were determined. Experimental protocols were approved by the Ethics Review Committee for Animal Experimentation at Handong Global University (Korea) (protocol \#HGU-2008-01).

\section{Conclusions}

In this study, the results of in vitro and in vivo analyses strongly indicated that zabofloxacin is very effective in the treatment of pneumonia caused by multi-drug-resistant S. pneumoniae, including QRSP. Overall, these results imply that zabofloxacin is a promising fluoroquinolone with potent activity against clinically isolated S. pneumoniae.

Acknowledgments: This research was supported by Dong Hwa Pharmaceutical Industry Ltd.

Author Contributions: Hee-Soo Park, Sang-Hun Oh and Hye Shin Kim designed and performed the experiments; Hee-Soo Park analyzed the data; Hee-Soo Park, and Jin-Hwan Kwak wrote the paper; and Jin-Hwan Kwak and Dong-Rack Choi supervised the study and helped interpret the results.

Conflicts of Interest: The authors declare no conflict of interest.

\section{References}

1. Varon, E.; Mainardi, J.L.; Gutmann, L. Streptococcus pneumoniae: Still a major pathogen. Clin. Microbiol. Infect. 2010, 16, 401. [CrossRef] [PubMed]

2. Levy, S.B.; Marshall, B. Antibacterial resistance worldwide: Causes, challenges and responses. Nat. Med. 2004, 10 (Suppl. 12), S122-S129. [CrossRef] [PubMed]

3. Ventola, C.L. The antibiotic resistance crisis: Part 1: Causes and threats. Pharm. Ther. 2015, 40, 277-283. [PubMed]

4. Kuster, S.P.; Rudnick, W.; Shigayeva, A.; Green, K.; Baqi, M.; Gold, W.L.; Lovinsky, R.; Muller, M.P.; Powis, J.E.; Rau, N.; et al. Previous antibiotic exposure and antimicrobial resistance in invasive pneumococcal disease: Results from prospective surveillance. Clin. Infect. Dis. 2014, 59, 944-952. [CrossRef] [PubMed]

5. Van Bambeke, F.; Reinert, R.R.; Appelbaum, P.C.; Tulkens, P.M.; Peetermans, W.E. Multidrug-resistant Streptococcus pneumoniae infections: Current and future therapeutic options. Drugs 2007, 67, 2355-2382. [CrossRef] [PubMed] 
6. Park, H.S.; Kim, H.J.; Seol, M.J.; Choi, D.R.; Choi, E.C.; Kwak, J.H. In vitro and in vivo antibacterial activities of DW-224a, a new fluoronaphthyridone. Antimicrob. Agents Chemother. 2006, 50, 2261-2264. [CrossRef] [PubMed]

7. Kwon, A.R.; Min, Y.H.; Ryu, J.M.; Choi, D.R.; Shim, M.J.; Choi, E.C. In vitro and in vivo activities of DW-224a, a novel fluoroquinolone antibiotic agent. J. Antimicrob. Chemother. 2006, 58, 684-688. [CrossRef] [PubMed]

8. Kim, T.; Park, S.J.; Chong, Y.P.; Park, K.H.; Lee, Y.M.; Hong, H.L.; Kim, H.S.; Kim, E.S.; Lee, S.; Choi, D.R.; et al. Fluoroquinolone resistance of Streptococcus pneumoniae isolates causing invasive disease: Special focus on zabofloxacin. Diagn. Microbiol. Infect. Dis. 2016, 86, 181-183. [CrossRef] [PubMed]

9. Kosowska-Shick, K.; Credito, K.; Pankuch, G.A.; Lin, G.; Bozdogan, B.; McGhee, P.; Dewasse, B.; Choi, D.R.; Ryu, J.M.; Appelbaum, P.C. Antipneumococcal activity of DW-224a, a new quinolone, compared to those of eight other agents. Antimicrob. Agents Chemother. 2006, 50, 2064-2071. [CrossRef] [PubMed]

10. Garvey, M.I.; Piddock, L.J. The efflux Pump Inhibitor Reserpine Selects Multidrug-resistant Streptococcus pneumoniae Strains That Overexpress the ABC transporters PatA and PatB. Antimicrob. Agents Chemother. 2008, 52, 1677-1685. [CrossRef] [PubMed]

11. Jones, R.N.; Biedenbach, D.J.; Ambrose, P.G.; Wikler, M.A. Zabofloxacin (DW-224a) activity against Neisseria gonorrhoeae including quinolone-resistant strains. Diagn. Microbiol. Infect. Dis. 2008, 62, 110-112. [CrossRef] [PubMed]

12. Kocsis, B.; Domokos, J.; Szabo, D. Chemical structure and pharmacokinetics of novel quinolone agents represented by avarofloxacin, delafloxacin, finafloxacin, zabofloxacin and nemonoxacin. Ann. Clin. Microb. Antimicrob. 2016, 15, 34. [CrossRef] [PubMed]

13. Kim, H.J.; Seol, M.J.; Park, H.S.; Choi, D.R.; Seong, S.K.; Shin, H.K.; Kwak, J.H. Antimicrobial activity of DW-224a, a new fluoroquinolone, against Streptococcus pneumoniae. J. Antimicrob. Chemother. 2006, 57, 1256-1258. [CrossRef] [PubMed]

14. Park, H.S.; Jung, S.J.; Kwak, J.H.; Choi, D.R.; Choi, E.C. DNA gyrase and topoisomerase IV are dual targets of zabofloxacin in Streptococcus pneumoniae. Int. J. Antimicrob. Agents 2010, 36, 97-98. [CrossRef] [PubMed]

15. Han, H.; Kim, S.E.; Shin, K.H.; Lim, C.; Lim, K.S.; Yu, K.S.; Cho, J.Y. Comparison of pharmacokinetics between new quinolone antibiotics: The zabofloxacin hydrochloride capsule and the zabofloxacin aspartate tablet. Curr. Med. Res. Opin. 2013, 29, 1349-1355. [CrossRef] [PubMed]

16. Clinical and Laboratory Standards Institute. Performance Standards for Antimicrobial Susceptibility Testing, Twenty-Third Informational Supplement M100-S23; Clinical and Laboratory Standards Institute: Wayne, PA, USA, 2013.

17. Brenwald, N.P.; Gill, M.J.; Wise, R. Prevalence of a putative efflux mechanism among fluoroquinoloneresistant clinical isolates of Streptococcus pneumoniae. Antimicrob. Agents Chemother. 1998, 42, 2032-2035. [PubMed]

18. Park, H.S.; Jung, S.J.; Choi, D.R.; Kwak, J.H. Antimicrobial activity of dw286 against Streptococcus pneumoniae. Int. J. Antimicrob. Agents 2010, 36, 230-233. [CrossRef] [PubMed]

19. Clinical and Laboratory Standards Institute. Methods for Determining Bactericidal Activity of Antimicrobial Agents: Approved Guideline M26-A; Clinical and Laboratory Standards Institute: Wayne, PA, USA, 1999.

20. Bliss, C.I. The Statistics of Bioassay; Academic Press, Inc.: New York, NY, USA, 1952.

Sample Availability: Not available.

(C) 2016 by the authors; licensee MDPI, Basel, Switzerland. This article is an open access article distributed under the terms and conditions of the Creative Commons Attribution (CC-BY) license (http://creativecommons.org/licenses/by/4.0/). 